\title{
Qualidade de vida de pacientes em tratamento com anticoagulante oral
}

\author{
Quality of life of patients treating with oral anticoagulant \\ Calidad de vida de los pacientes que tratan con anticoagulante oral
}

Hirla Vanessa Soares de Araújo ${ }^{1 *}$, Andreza do Carmo de Albuquerque Pôrto Meira², Paulo Henrique do Nascimento Bem², Rita de Cássia Cordeiro Bastos Leite dos Anjos ${ }^{2}$, Rebeka Maria de Oliveira Belo로 Simone Maria Muniz da Silva Bezerra ${ }^{1}$, Maria Regina Silva de Saturno².

\section{RESUMO}

Objetivo: Avaliar a qualidade de vida de pacientes que fazem uso de anticoagulante oral. Métodos: Estudo descritivo, transversal, com abordagem quantitativa, realizado no ambulatório egresso de anticoagulante oral de um hospital de referência. A amostra foi de 93 pacientes. Foi utilizado questionário semiestruturado, abordando variáveis socioeconômicas e clínicas e o instrumento Duke Anticoagulation Satisfaction Scale (DASS). Resultados: Houve predominância do sexo feminino $(51 ; 54,4 \%)$, com idade média de $51,25 \pm 12,10$ anos. A principal indicação de anticoagulante oral foi a troca de válvula cardíaca $(46 ; 49,5 \%)$ seguida da fibrilação atrial $(18 ; 19 \%)$. Na avaliação da qualidade de vida, no domínio total o resultado encontrado foi de $79,0 \pm 21,6$ indicando que estes usuários apresentam pior qualidade de vida quando comparados com a literatura. Conclusão: Este estudo demonstrou que os pacientes apresentaram baixa qualidade de vida. Observou-se que quanto maior a idade do indivíduo, menor o escore DASS e melhor qualidade de vida. As comorbidades associadas são fatores que contribuem para uma pior avaliação.

Palavras-chave: Qualidade de vida, Anticoagulantes, Doenças cardiovasculares, Cardiologia, Cuidados de enfermagem.

\begin{abstract}
Objective: To assess the quality of life of patients using oral anticoagulants. Methods: A descriptive, crosssectional study, with a quantitative approach, carried out in the outpatient clinic of anticoagulants at a referral hospital. The sample consisted of 93 patients. A semi-structured questionnaire was used, addressing socioeconomic and clinical variables and the Duke Anticoagulation Satisfaction Scale (DASS) instrument. Results: There was a predominance of females $(51 ; 54.4 \%)$, with a mean age of $51.25 \pm 12.10$ years. The main indication for oral anticoagulants was cardiac valve replacement $(46 ; 49.5 \%)$ followed by atrial fibrillation (18; $19 \%)$. In the assessment of quality of life, in the total domain, the result found was $79.0 \pm 21.6$ indicating that these users have a worse quality of life when compared to the literature. Conclusion: This study demonstrated that patients had low quality of life. It was observed that the older the individual, the lower the DASS score and the better quality of life. Associated comorbidities are factors that contribute to a worse assessment.
\end{abstract}

Key words: Quality of life, Anticoagulants, Cardiovascular disease, Cardiology, Nursing care.

\section{RESUMEN}

Objetivo: Evaluar la calidad de vida de los pacientes que utilizan anticoagulantes orales. Métodos: Estudio descriptivo, transversal, con abordaje cuantitativo, realizado en la consulta externa de anticoagulantes de un hospital de referencia. La muestra estuvo formada por 93 pacientes. Se utilizó un cuestionario

1 Universidade de Pernambuco (UPE), Recife - PE. *E-mail: hirlavs.araujo@gmail.com

2 Hospital Dom Helder Câmara, Cabo de Santo Agostinho - PE. 
semiestructurado, abordando variables socioeconómicas y clínicas, y el instrumento Duke Anticoagulation Satisfaction Scale (DASS). Resultados: Predominó el sexo femenino $(51 ; 54,4 \%)$, con una edad media de $51,25 \pm 12,10$ años. La principal indicación de los anticoagulantes orales fue el reemplazo valvular cardíaco (46; $49,5 \%)$ seguido de la fibrilación auricular (18; 19\%). En la evaluación de la calidad de vida, en el dominio total, el resultado encontrado fue $79.0 \pm 21.6$ indicando que estos usuarios tienen una peor calidad de vida en comparación con la literatura. Conclusión: Este estudio demostró que los pacientes tenían baja calidad de vida. Se observó que a mayor edad, menor puntuación DASS y mejor calidad de vida. Las comorbilidades asociadas son factores que contribuyen a una peor valoración.

Palabras clave: Calidad de vida, Anticoagulantes, Enfermedades cardiovasculares, Cardiología, Atención de enfermería.

\section{INTRODUÇÃO}

As doenças crônicas não transmissíveis são consideradas problema de saúde pública, não só no Brasil, como na América, contribuindo para agravos à população. Dentre as doenças crônicas, destacam-se as doenças cardiovasculares, sendo consideradas as primeiras causas de óbito no país (MALTA DC, et al., 2020; DUTRA DD, et al., 2016).

Para algumas dessas doenças há indicação para uso de Anticoagulantes Orais (ACO). Trata-se de uma medicação amplamente estabelecida no que diz respeito à sua eficácia e segurança no tratamento. Para tanto, se faz necessária a monitorização periódica da coagulação sanguínea do paciente, em especial de pacientes que fazem de medicamentos antivitamina K (SOUZA TF, et al., 2018; SIMONETTI SH, et al., 2019).

Possuindo uma janela terapêutica estreita e a estabilidade da anticoagulação com aspecto multifatorial, o tratamento requer acompanhamento em longo prazo para monitorar os níveis de anticoagulação dos pacientes e minimizar a incidência de efeitos adversos tromboembólicos ou hemorrágicos (LEAL PM, et al., 2020; NISHIMURA RA, et al., 2014).

A ação terapêutica do ACO pode ser influenciada por numerosas condições que alteram a sua absorção e metabolismo. Como exemplos, podem ser citados a ingestão de alimentos ricos em gorduras e vitamina $\mathrm{K}$, medicamentos contendo ácido acetil salicílico e bebidas alcoólicas (TARASOUTCHI F, et al., 2017). Dessa forma, o controle e manutenção da coagulação sanguínea ideal exigem dos usuários de ACO visitas constantes aos serviços de saúde bem como mudanças nos hábitos de vida que repercutir na qualidade de vida relacionada à saúde do indivíduo em terapia anticoagulante.

Segundo a Organização Mundial da Saúde, a qualidade de vida apresenta um conceito amplo e subjetivo que considera a percepção do indivíduo sobre o contexto no qual se encontra inserido (WHOQOL, 1995). No entanto, quando a qualidade de vida passa a sofrer influência de condições clínicas de saúde, relacionandose com o processo saúde-doença, passa a ser conhecida como a qualidade de vida relacionada à saúde (QVRS).

A QVRS pode ser definida como o valor atribuído à duração da vida quando modificada pela percepção de limitações físicas, psicológicas, funções sociais e, ainda, oportunidades influenciadas pela doença, sendo o paciente a melhor fonte de informação sobre sua qualidade de vida. Dessa forma, a QVRS é reconhecida como sendo uma questão de importância e que preocupa os profissionais de enfermagem (ARAÚJO HVS, et al., 2017). Diante da literatura revisada, percebe-se a importância de realizar estudos com o objetivo de avaliar a qualidade de vida dos pacientes que fazem uso de anticoagulante oral e sua relação com o perfil clínico e socioeconômico (ALMEIDA NETO, et al., 2016).

Com a finalidade de conscientizar aos profissionais enfermeiros sobre a importância de melhorar a qualidade de vida dos pacientes anticoagulados e buscar medidas de educação em saúde, promoção da saúde e prevenção de agravos, o objetivo desta pesquisa foi avaliar a qualidade de vida de pacientes que fazem uso de ACO. 


\section{MÉTODOS}

Trata-se de um estudo descritivo, transversal, com abordagem quantitativa. O estudo foi realizado no ambulatório egresso de anticoagulante oral de um Hospital de referência em cardiologia da Região metropolitana de Recife, localizado na cidade de Cabo de Santo Agostinho, Pernambuco.

A população compreendeu pacientes cardiopatas em uso de anticoagulantes orais. O cálculo da amostra foi determinado para populações finitas, considerando um $\mathrm{N}$ de 245 pacientes, considerando um intervalo de confiança de $95 \%$ e um erro amostral de $5 \%$. A amostra calculada foi de 149 pacientes, no entanto, houve perdas durante a coleta dos dados, levando à uma amostra final de 93 pacientes.

Foram incluídos pacientes com idade igual ou maior a 18 anos, em tratamento com anticoagulantes orais, acompanhados no ambulatório onde seria realizada a pesquisa e em seguimento ambulatorial há pelo menos três meses. Foram excluídos pacientes com déficit cognitivo que impediu a compreensão do estudo e aplicação dos questionários.

O período da coleta de dados foi de maio a outubro de 2019. Para a coleta dos dados foram necessários dois questionários. O primeiro, um questionário semiestruturado foi elaborado pelas pesquisadoras do estudo, abordam as variáveis socioeconômicas e clínicas.

A qualidade de vida dos usuários de anticoagulante oral foi avaliada por meio do instrumento Duke Anticoagulation Satisfaction Scale (DASS). Trata-se de um instrumento que perpassou pelo processo de adaptação cultural e validação para uso no Brasil em usuários de $A C O$, contendo 25 itens referentes às limitações das atividades diárias pelo uso de varfarina. As opções de respostas apresentam uma escala de 0 a 7 pontos (PELEGRINO FM, 2009).

O instrumento é subdividido em três domínios, a saber: Limitação, Tarefa/Sobrecarga e Impacto Psicológico. A soma dos domínios resulta no escore total do DASS, com variação de 25 a 175 pontos na escala. Valores mais altos representam pior satisfação e qualidade de vida do paciente em relação ao uso de ACO e maiores limitações (PELEGRINO FM, 2009).

Os itens pertencentes às dimensões tarefas/sobrecarga (item 3h) e impacto psicológico (4a, 4b, 4F, 4H e 4j) foram analisados de forma reversa para manter a consistência, pois nesses itens, os menores escores indicam maior satisfação (PELEGRINO FM, 2009).

Os dados quantitativos obtidos foram categorizados e processados eletronicamente através do programa base de dados Microsoft Excel 2010 na forma de tabelas e expressos por frequências absolutas e relativas (porcentagens). Foram calculadas médias e desvio padrão, analisados no software SPSS 20.0. Foi realizado o teste de normalidade das variáveis, sendo de distribuição não normal. Logo, o teste estatístico para comparação de médias utilizado foi o não paramétrico Mann Whitney.

Os participantes assinaram o Termo de Consentimento Livre e Esclarecido em duas vias após os esclarecimentos. A referida pesquisa foi realizada a partir do preconizado na Resolução 466/2012, do Conselho Nacional de Saúde. O projeto ao qual o presente estudo está vinculado foi aprovado pelo Comitê de Ética com parecer n³.226.877 e CAAE 07938119.1.0000.5201 em 27/03/2019.

\section{RESULTADOS}

No período do estudo foram entrevistados 93 usuários em uso de anticoagulantes orais que estavam em seguimento ambulatorial, sendo a média de idade de $51,25( \pm 12,10)$ anos, maioria do sexo feminino $(54,4 \%)$, pardos e negros $(66,7 \%)$, exercendo atividade laboral $(52,6 \%)$. Também foram predominantes pacientes que possuem companheiro (68,8\%), que seguem doutrina religiosa $(91,4 \%)$ e que apresentam baixo nível de escolaridade (64,5\%). Ainda, $81,7 \%$ dos pacientes apresentam baixa renda, 69,9\% utilizam transporte público para acessar o serviço de saúde e $67,7 \%$ moram na zona urbana (Tabela 1). Devem se limitar a descrever os resultados encontrados, sem incluir interpretações e/ou comparações. O texto deve complementar e não repetir o que está descrito nas figuras. 
Tabela 1 - Caracterização social e demográfica dos pacientes, $n=93$.

\begin{tabular}{|c|c|c|}
\hline Variáveis sociais e demográficas & Média & $( \pm d p)$ \\
\hline \multirow[t]{2}{*}{ Idade } & 51,25 & $\pm 12,10$ \\
\hline & $\mathbf{n}$ & $(\%)$ \\
\hline \multicolumn{3}{|l|}{ Etnia } \\
\hline Branca & 31 & 33,3 \\
\hline Pardos e negros & 62 & 66,7 \\
\hline \multicolumn{3}{|l|}{ Sexo } \\
\hline Feminino & 51 & 54,8 \\
\hline Masculino & 42 & 45,2 \\
\hline \multicolumn{3}{|l|}{ Ocupação } \\
\hline Sem atividade laboral & 49 & 52,6 \\
\hline Com atividade laboral & 44 & 47,4 \\
\hline \multicolumn{3}{|l|}{ Estado civil } \\
\hline Com companheiro & 64 & 68,8 \\
\hline Sem companheiro & 29 & 31,2 \\
\hline \multicolumn{3}{|l|}{ Religião } \\
\hline Tem doutrina religiosa & 85 & 91,4 \\
\hline Não tem doutrina religiosa & 8 & 8,6 \\
\hline \multicolumn{3}{|l|}{ Escolaridade } \\
\hline Até 9 anos de estudos & 60 & 64,5 \\
\hline Mais de 9 anos e estudos & 33 & 35,5 \\
\hline Reside sozinho & 22 & 23,7 \\
\hline \multicolumn{3}{|l|}{ Renda Mensal } \\
\hline Até 1 salário mínimo & 17 & 18,3 \\
\hline Mais de um salário mínimo & 76 & 81,7 \\
\hline \multicolumn{3}{|l|}{ Transporte } \\
\hline Público & 65 & 69,9 \\
\hline Particular & 28 & 30,1 \\
\hline \multicolumn{3}{|l|}{ Moradia } \\
\hline Zona Rural & 30 & 32,3 \\
\hline Zona Urbana & 63 & 67,7 \\
\hline
\end{tabular}

Fonte: Araújo HVS, et al., 2021.

Quanto à caracterização dos indivíduos segundo a terapia de anticoagulantes orais constatou-se que a principal indicação para o tratamento ambulatorial foi a troca de válvula $(49,5 \%)$ seguida da fibrilação atrial $(19 \%)$ e outras causas (13\%). Ainda, $28 \%$ dos pacientes apresentavam febre reumática como comorbidade associada, sendo que destes, $27 \%$ tiveram necessidade de cirurgia (Tabela 2).

Em relação ao tempo de tratamento desses pacientes, a maioria apresentava mais de 6 meses $(83,9 \%)$. Durante o período de tratamento, $30,1 \%$ dos pacientes relataram que apresentaram eventos adversos, tendo como principal evento o sangramento (30,1\%). Não obstante, $29 \%$ dos pacientes apresentaram a necessidade de internação em algum momento do tratamento devido ao uso do medicamento.

As comorbidades mais frequentes foram a hipertensão arterial sistêmica presente em $76,3 \%$ dos pacientes, seguida de $63,4 \%$ da fibrilação atrial, $23,7 \%$ infarto agudo do miocárdio e outras. Cerca de 59,1\% dos pacientes apresentavam história familiar de cardiopatia, 4,3\% tabagismo e 8,6\% uso de bebida alcoólica. 
Tabela 2 - Características clínica dos pacientes atendidos no ambulatório de anticoagulação oral, n=93.

\begin{tabular}{lcc}
\hline Variáveis clínicas & $\mathbf{n}$ & $(\%)$ \\
\hline Indicação & 18 & 19 \\
FA & 11 & 12 \\
ICC & 46 & 49,5 \\
Troca de válvula & 6 & 6,5 \\
Doença coronária & 12 & 13 \\
Outras & & 16,1 \\
\hline Tempo de tratamento & 15 & 83,9 \\
\hline Até 6 meses & 78 & 50,5 \\
Mais de 6 meses & & 30,1 \\
\hline Eventos adversos & 47 & 19,4 \\
\hline Não apresentou & 28 & 29 \\
Sangramento & 18 & 63,4 \\
Outros & 27 & 59,1 \\
\hline Necessidade de Internamento & & 76,3 \\
\hline Comorbidades & 59 & 16,1 \\
Fibrilação Atrial & 55 & 22,6 \\
História Familiar de Cardiopatia & 71 & 23,7 \\
Hipertensão Arterial Sistêmica & 15 & 2,2 \\
Diabetes Mellitus & 21 & 49,5 \\
Acidente Vascular Encefálico & 22 & 4,3 \\
Infarto Agudo do Miocárdio & 2 & 8,6 \\
Cirurgia de Revascularização & 46 & \\
Cirurgia de Troca Valvar & 4 & 8 \\
Tabagismo & & \\
Uso de álcool & & \\
\hline Fonte: Araújo HVS, et al., 2021. & & \\
\hline
\end{tabular}

Fonte: Araújo HVS, et al., 2021.

A qualidade de vida é um fator importante de ser observado em pacientes anticoagulados e estes dados estão descritos abaixo. Em relação ao Escore Total, a média foi de $79,0( \pm 21,6)$, com uma pontuação que variou de 33 a 125. No domínio Impacto Psicológico a média foi de $23,4( \pm 5,6)$, com pontuação que vaiou de 10 a 41 pontos. No instrumento de avaliação DASS o domínio Impacto Psicológico é subdivido em Impacto Positivo e Impacto Negativo, conforme apresentado na tabela. Em relação ao domínio Tarefa e Sobrecarga a média foi de $25,4( \pm 10,8)$. Neste domínio os valores variaram entre 8 a 52 pontos. Por fim, o domínio Limitações apresentou uma média de 30,2 $( \pm 11,7)$, tendo sua pontuação mínima e máxima entre 9 a 54 pontos, respectivamente (Tabela 3 ).

Tabela 3 - Avaliação da Qualidade de Vida Relacionada à Saúde pelo Duke anticoagulation de pacientes em uso de anticoagulantes orais, $n=93$.

\begin{tabular}{lcccc}
\hline Escala de Satisfação da Anticoagulação de DUKE/ Intervalos & Média & DP & Mín & Máx \\
\hline Escore Total (25-175) & 79,0 & $\pm 21,6$ & 33 & 125 \\
Limitações (9-63) & 30,2 & $\pm 11,7$ & 9 & 54 \\
Tarefa/Sobrecarga (8-56) & 25,4 & $\pm 10,8$ & 8 & 52 \\
Impacto Psicológico (8-56) & 23,4 & $\pm 5,6$ & 10 & 41 \\
Positivo (5-35) & 11,9 & $\pm 4,1$ & 5 & 26 \\
Negativo (3-21) & 11,5 & $\pm 4,2$ & 3 & 21 \\
\hline
\end{tabular}

Fonte: Araújo HVS, et al., 2021.

Em relação à contribuição de cada resposta na avaliação por domínio, no que tange ao domínio Limitações os entrevistados foram questionados sobre o quanto sua alimentação mudou devido ao tratamento com o anticoagulante, no qual, 59 \% dos usuários relataram mudar muito ou muitíssimo sua alimentação devido ao ACO.

Em relação ao domínio Tarefa e Sobrecarga, no que diz respeito a dificuldade em seguir o tratamento, $33 \%$ dos entrevistados acreditavam ser bastante, muito ou muitíssimo difícil seguir tratamento com a varfarina. Ainda nessa avaliação, $40 \%$ dos usuários não relataram nenhuma dificuldade em seguir este tratamento, $13 \%$ relataram dificuldade moderada, $10 \%$ pouca dificuldade e $4 \%$ muito pouca dificuldade. Observou-se também que $73 \%$ dos usuários acreditam conseguir aderir corretamente ao tratamento, seguido de $22 \%$ que acreditam seguir mais ou menos e de $5 \%$ que acreditam seguir pouco. 
Sobre as perguntas que abrangem o impacto psicológico causado pelo uso de ACO, em relação a compreensão do motivo para o uso deste medicamento, $54 \%$ referiram compreender de bastante a muitíssimo, $20 \%$ declararam mais ou menos e $26 \%$ declararam compreender muito pouco. Neste domínio estava presente a pergunta que apresentou uma média com maior destaque de todo questionário: o quanto que o usuário se preocupava com o risco de sangramento ou de se machucar. Nesta avaliação houve uma média de 5,8 pontos, representando, desta forma, um questionamento de grande impacto na qualidade de vida do paciente.

Os valores para impacto psicológico positivo foram baixos. Este resultado indica boa satisfação e compreensão em relação ao tratamento. Em contrapartida, os valores para o domínio de impacto psicológico negativo foram altos, resultado que indica que os usuários são muito afetados, de forma negativa, pelo uso da medicação.

Uma outra avaliação permitida pelo instrumento foi a de verificação das médias do DASS nos pacientes que necessitaram ou não de alguma internação durante o tratamento com a medicação em questão. Logo $29 \%$ dos pacientes necessitaram de internação devido a alguma complicação com o uso do ACO, onde a média do escore do DASS foi de 80,3 ( \pm 22 ). Já os pacientes que não necessitaram de internações, a média foi de 78,4 ( $\pm 21,6)$, uma diferença mínima entre os grupos, não apresentando, desta forma, grande diferença no que se refere a qualidade de vida.

Em relação a avaliação das variáveis sociodemográficas e clínicas em função da qualidade de vida, podese observar significância estatística (Tabela 4). Em relação à idade, pacientes com menos de 60 anos apresentaram pior avaliação da qualidade de vida no domínio Limitações $(p=0,022)$. No que diz respeito à ocupação, pacientes com atividades laborais tiveram pior avaliação no Escore Total $(p=0,014)$ do instrumento e no domínio Limitações $(p=0,009)$.

No domínio Impacto Psicológico Negativo, pacientes de baixa escolaridade tiveram pior avaliação para o domínio (0,018). Em relação as comorbidades, pacientes com fibrilação atrial tiveram melhor avaliação no domínio Impacto Psicológico Positivo. Já os pacientes com hipertensão tiveram pior avaliação no domínio Impacto Psicológico $(p=0.033)$.

Na avaliação do Impacto Psicológico Positivo, pacientes hipertensões tiveram pior avaliação da qualidade de vida $(p=0,019)$. Por fim, pacientes que realizaram cirurgia de troca valvar tiveram melhor avaliação da qualidade de vida no domínio Impacto Psicológico Positivo $(p=0,044)$.

Tabela 4 - Avaliação da QVRS em função de variáveis sociodemográficas e de saúde, n=93.

\begin{tabular}{|c|c|c|c|c|c|c|c|c|c|c|c|c|}
\hline \multirow{2}{*}{$\begin{array}{l}\text { Variável/ } \\
\text { Domínios }\end{array}$} & \multicolumn{2}{|c|}{ Escore Total } & \multicolumn{2}{|c|}{ Limitações } & \multicolumn{2}{|c|}{$\begin{array}{c}\text { Tarefas/ } \\
\text { Sobrecarga }\end{array}$} & \multicolumn{2}{|c|}{ Psicológico } & \multicolumn{2}{|c|}{$\begin{array}{l}\text { Psicológico } \\
\text { Positivo }\end{array}$} & \multicolumn{2}{|c|}{$\begin{array}{l}\text { Psicológico } \\
\text { negativo }\end{array}$} \\
\hline & $\mathbf{M}^{*}$ & $\mathbf{p}^{\star \star}$ & $\overline{\mathbf{M}^{*}}$ & $\mathbf{p}^{\star \star}$ & $\mathbf{M}^{*}$ & $p^{\star \star}$ & $\mathbf{M}^{*}$ & $\mathbf{p}^{\star \star}$ & $\mathbf{M}^{*}$ & $\mathbf{p}^{\star \star}$ & $\mathbf{M}^{*}$ & $\mathbf{p}^{\star \star}$ \\
\hline $\begin{array}{l}\text { Idade }<60 \text { anos } \\
\text { Idade }>60 \text { anos }\end{array}$ & $\begin{array}{l}83,00 \\
67,00\end{array}$ & 087, & $\begin{array}{l}33,00 \\
22,00\end{array}$ & ,022 & $\begin{array}{l}26,00 \\
21,00\end{array}$ & 461, & $\begin{array}{l}24,00 \\
21,50\end{array}$ & 576, & $\begin{array}{l}12,00 \\
12,00\end{array}$ & 151, & $\begin{array}{l}4,00 \\
3,33\end{array}$ & 057, \\
\hline $\begin{array}{l}\text { Com ocupação } \\
\text { Sem ocupação }\end{array}$ & $\begin{array}{l}87,00 \\
71,50\end{array}$ & ,014 & $\begin{array}{l}35,00 \\
24,50\end{array}$ & ,009 & $\begin{array}{l}28,00 \\
21,50\end{array}$ & 148, & $\begin{array}{l}23,00 \\
23,00\end{array}$ & 874, & $\begin{array}{l}2,20 \\
2,40\end{array}$ & 412, & $\begin{array}{l}3,67 \\
4,00\end{array}$ & 291, \\
\hline $\begin{array}{l}<9 \text { anos de } \\
\text { estudo } \\
>9 \text { anos de } \\
\text { estudo }\end{array}$ & $\begin{array}{l}73,00 \\
84,00\end{array}$ & 223, & $\begin{array}{l}31,00 \\
31,00\end{array}$ & 645, & $\begin{array}{l}22,00 \\
26,00\end{array}$ & 194, & $\begin{array}{l}23,00 \\
24,00\end{array}$ & 353, & $\begin{array}{l}12,00 \\
11,00\end{array}$ & 228, & $\begin{array}{l}10,00 \\
13,00\end{array}$ & 018, \\
\hline $\begin{array}{l}\text { Com FA } \\
\text { Sem FA }\end{array}$ & $\begin{array}{l}83,00 \\
75,00\end{array}$ & 167, & $\begin{array}{l}31,00 \\
30,50\end{array}$ & 4 & $\begin{array}{l}26,00 \\
24,50\end{array}$ & 095, & $\begin{array}{l}24,00 \\
20,50\end{array}$ & 073, & $\begin{array}{l}12,00 \\
10,00\end{array}$ & 039, & $\begin{array}{l}12,00 \\
10,50\end{array}$ & 419, \\
\hline $\begin{array}{l}\text { Com HAS } \\
\text { Sem HAS }\end{array}$ & $\begin{array}{l}83,00 \\
75,00\end{array}$ & 478, & $\begin{array}{l}31,00 \\
30,50\end{array}$ & ,772 & $\begin{array}{l}26,00 \\
24,50\end{array}$ & 758, & $\begin{array}{l}24,00 \\
20,50\end{array}$ & 033, & $\begin{array}{c}12,00 \\
9,50\end{array}$ & ,019, & $\begin{array}{l}11,00 \\
11,00\end{array}$ & 602, \\
\hline $\begin{array}{l}\text { Realizou CTV } \\
\text { Não realizou CTV }\end{array}$ & $\begin{array}{l}79,00 \\
83,00\end{array}$ & 465, & $\begin{array}{l}31,00 \\
31,00\end{array}$ & 275, & $\begin{array}{l}26,00 \\
23,00\end{array}$ & 150, & $\begin{array}{l}23,00 \\
24,00\end{array}$ & 168, & $\begin{array}{l}11,00 \\
13,00\end{array}$ & ,044 & $\begin{array}{l}11,00 \\
11,00\end{array}$ & 826, \\
\hline
\end{tabular}

Legenda: *Mediana; ${ }^{\star *}$ Teste Mann Whitney; FA: fibrilação atrial; HAS: hipertensão arterial sistêmica; CTV: cirurgia de troca valvar.

Fonte: Araújo HVS, et al., 2021. 


\section{DISCUSSÃO}

O estudo permitiu obter informações importantes sobre aspectos que refletem a complexidade do tratamento com anticoagulantes orais e o reflexo dele na qualidade de vida dos usuários.

A média de idade dos participantes foi de 51,25 anos, resultado semelhante às pesquisas nacionais realizadas com pacientes em uso de anticoagulantes e em acompanhamento ambulatorial especializado (FIGUEIRÊDO TR, et al., 2017).

Em relação ao sexo, verificou-se maior participação de mulheres em uso de anticoagulante oral, resultado semelhante ao encontrado em outro estudo que identificou o perfil de saúde de pacientes em uso de ACO (SOUZA TF, et al., 2018; FIGUEIRÊDO TR, et al., 2017), demonstrando uma realidade comportamental constante e naturalizada, alicerçada em uma perspectiva cultural, na qual as mulheres têm mais percepção das doenças, bem como maior preocupação com a saúde (DUTRA DD, et al., 2016). Em contrapartida alguns estudos verificaram uma maior frequência de homens entre os usuários de anticoagulantes (DALPIAZ J, et al., 2017; ALMEIDA NETO et al., 2016).

Em relação ao estado civil, a maioria dos usuários apresentam companheiros, corroborando com outros estudos realizados que também tinham como objetivo descrever o perfil de usuários de ACO (FIGUEIRÊDO TR, et al., 2017; ALMEIDA NETO et al., 2016). Portanto, o suporte familiar, influencia positivamente o paciente acometido com doença crônica, sendo um importante aliado ao tratamento (BROTTO AM e GUIMARAES ABP, 2017).

No tocante aos anos de estudo, a maioria dos usuários apresentaram baixo nível de escolaridade resultado também predominante em outro estudo realizado no serviço público que atende pacientes em uso de ACO (FIGUEIRÊDO TR, et al., 2017; DALPIAZ J, et al., 2017). Estudos epidemiológicos sobre doenças crônicas inferem que estas ocorrem com maior frequência em populações economicamente desfavoráveis e de baixa escolaridade (DALPIAZ J, et al., 2017; ALMEIDA NETO et al., 2016). Diante deste cenário, sabe-se que o nível de escolaridade baixo pode impactar de forma negativa na adesão ao tratamento e, consequentemente, interferir na qualidade de vida, pois aqueles com menor grau de instrução demonstram ter menor conhecimento em relação ao tratamento quando comparados a usuários com maior grau de escolaridade (FIGUEIRÊDO TR, et al., 2017).

As principais indicações para o uso de ACO foram a troca valvar, seguida da fibrilação atrial e outras indicações, resultado semelhante ao encontrado em outro estudo que busca descrever o perfil sociodemográfico e clínico dos pacientes em uso de ACOS, as valvopatias e a fibrilação atrial foram igualmente frequentes, apresentando-se tanto como condição principal de tratamento, quanto associadas em um mesmo indivíduo (FIGUEIRÊDO TR, et al., 2017). Segundo estudo as principais indicações para o uso de terapia anticoagulante são embolia pulmonar, trombose, paciente com prótese valvar cardíaca, síndromes coronarianas, dentre outras indicações (SILVA TE, et al., 2019).

Diante dos achados encontrados alguns estudos demonstram a alta prevalência destas afecções e a necessidade de otimização do tratamento com os anticoagulantes orais, a fim de diminuir o número de complicações hemorrágicas e tromboembólicas relacionadas à terapia (FERREIRA J e MIRCO A, 2015).

Quanto as comorbidades associadas, a hipertensão arterial apresentou grande destaque, além da Fibrilação Atrial, Infarto Agudo do Miocárdio e Acidente Vascular Encefálico, corroborando com outro estudo (FIGUEIRÊDO TR, et al., 2017). Comorbidades associadas, incluindo a presença de fibrilação atrial, representam fator de risco maior para a ocorrência de outras doenças cardiovasculares, como o Acidente Vascular Encefálico (STAMBLER B e SCAZZUSO F, 2016).

Em relação à avaliação da qualidade de vida, O DASS é um instrumento que tem como objetivo quantificar a satisfação e a qualidade de vida pelo uso de anticoagulante orais. No que diz respeito ao Escore Total, na presente pesquisa os pacientes apresentaram pior avaliação da qualidade de vida em comparação com outras pesquisas. Um estudo realizado em um ambulatório de anticoagulação de um hospital de São Paulo, a média do Escore Total foi de 57,6 (PELEGRINO FM, 2009). 
Em um outro estudo realizado em três instituições hospitalares de Cascavel/PR, que avaliou a qualidade de vida e adesão dos seis primeiros meses de tratamento obteve uma média de 45,1 pontos para o DASS (PELEGRINO FM, 2009). Já em um outro estudo que tem como finalidade avaliar a qualidade de vida de usuários de ACO no município de ljuí/RS, a média foi de 71,85 (DALPIAZ J, et al., 2017).

No tocante ao Escore Total e o domínio Limitações, pode-se observar relação estatisticamente significativa entre os usuários que apresentava ou não ocupação, interferindo diretamente na qualidade de vida desses pacientes, pois apesar da maioria não exercer atividades laborais, os usuários que possuíam alguma ocupação apresentaram pior qualidade de vida. Esse resultado, semelhante ao de um estudo que traça o perfil dos usuários que fazem uso do ACO (FIGUEIRÊDO TR, et al., 2017), pode ser justificado pelas limitações físicas impostas pela doença.

No que se refere ao domínio Limitações, foi o que apresentou maior impacto nos usuários, divergindo de outras pesquisas, nas quais esse domínio apresentou menor média e impacto para a população (DALPIAZ J, et al., 2017). As restrições relacionadas a alimentação, são significativas para a maioria dos usuários desse estudo, o que vai de encontro com a pesquisa na qual para $49 \%$ dos usuários essa pergunta não se aplicou, pois estes não tiveram nenhum tipo de mudança alimentar relacionado ao uso do ACO cumarínicos (DALPIAZ J, et al., 2017).

Diversas mudanças nos hábitos de vida de pacientes que fazem uso de ACO cumarínicos devem ser instituídas, tais como adequação no padrão alimentar, redução da ingestão de alimentos ricos em gorduras e alimentos ricos em vitamina $\mathrm{K}$. Desta forma, ressalta-se a importância de orientar os usuários a manter uma ingesta regular de alimentos ricos em vitamina $\mathrm{K}$, uma vez que o hábito pode auxiliar na estabilidade do INR (CHANG CH et al., 2014).

Em relação à idade, no que se refere a associação da mesma com o domínio Limitações, observou-se que quanto menor a idade pior a avaliação da qualidade de vida dos usuários entrevistados. Este resultado corrobora com o estudo que avalia a qualidade de vida de portadores de prótese valvar mecânica cardíaca, no qual quanto maior idade, maior percepção da sua qualidade de vida (MALTA MM, 2015).

O domínio relacionado a Tarefas e Sobrecargas, contempla perguntas como adesão, satisfação e as dificuldades com o tratamento. Esse domínio foi o segundo com menor impacto na qualidade de vida dos usuários, esses resultados refletem pouca dificuldade e grande adesão ao tratamento, sendo esse achado semelhante ao encontrado na pesquisa realizada em um serviço público de ljuí (DALPIAZ J, et al., 2017).

Os menores resultados de impacto do tratamento com ACO foram obtidos no domínio psicológico, ainda que maiores quando comparados a outro estudo (PELEGRINO FM, 2009). Desta forma, o resultado implica em comprometimento na qualidade de vida bem como na satisfação e compreensão do paciente quanto à necessidade do tratamento.

Ainda no que tange ao Impacto Psicológico, este domínio é subdividido em Impacto Psicológico Positivo e Impacto Psicológico Negativo. Os resultados mostram que os valores obtidos para o Impacto Psicológico Negativo foram maiores do que os encontrados em outro estudo, indicando que os usuários são afetados negativamente pelo uso do ACO. Um questionamento do DASS que pode ter contribuído para tal resultado está relacionado com o risco de apresentar sangramento ou de machucar, apesar da maioria nunca ter apresentado algum evento adverso ao ACO.

No que se refere aos impactos psicológicos positivos pode-se perceber que os pacientes que apresentaram comorbidades associadas como FA e HAS apresentaram menor qualidade de vida ao comparado com os usuários que não possuem comorbidades associadas. Estudo sobre avaliação da qualidade de vida em idosos com fibrilação atrial verificou que pacientes medicados com os novos anticoagulantes orais apresentaram melhor qualidade de vida no domínio psicológico positivo. Esse resultado pode ser justificado pelo motivo de que os pacientes que usam os novos anticoagulantes não necessitam da peridiocidade de monitorização da coagulação quando comparado com os ACO antivitamina K (MORAIS Al, et al., 2020). Sendo justificado através de estudos que após substituição da valva cardíaca os pacientes apresentam estabilidade da anticoagulação fazendo com que os pacientes tenham uma melhor satisfação com a anticoagulação oral e qualidade de vida (MALTA MM, 2015). 
Estes achados confirmam estudos que demonstram a alta prevalência destas afecções e a necessidade de otimização do tratamento com os anticoagulantes orais, a fim de reduzir significativamente o número de complicações tromboembólicas (FERREIRA J e MIRCO A, 2015).

\section{CONCLUSÃO}

Este estudo demonstrou que os pacientes apresentaram baixa qualidade de vida. Observou-se que quanto maior a idade do indivíduo, menor o escore DASS e melhor qualidade de vida. E que as comorbidades associadas são fatores que contribuem para uma pior avaliação. As questões que envolvem a QVRS de pacientes em uso de ACO possibilitaram a reflexão sobre a real necessidade do paciente anticoagulado em relação a sua condição de saúde, qualidade de vida e satisfação pessoal, o que pode, diretamente, auxiliar os profissionais de saúde envolvidos no planejamento de reabilitação. Como consequência deste estudo, foi possível identificar particularidade e aspectos relevantes a serem evidenciados no momento da prestação de cuidados e assistência à saúde de pacientes em terapia com ACO's.

\section{REFERÊNCIAS}

1. ALMEIDA NETO OP, et al. Perfil clínico, adesão e satisfação terapêutica de pacientes em uso de anticoagulantes orais. Rev Aten Saúde. 2016; 14(47) :61-6.

2. ARAÚJO HVS, et al. Quality of life of patients who undergone myocardial revascularization surgery. Rev Bras Enferm. 2017; 70(2): 257-64

3. BROTTO AM, GUIMARAES ABP. A influência da família no tratamento de pacientes com doenças crônicas. Psicol. hosp. (São Paulo) [online]. 2017; 15(1): 43-68.

4. $\mathrm{CHANG} \mathrm{CH}$, et al. A practical approach to minimize the interaction of dietary vitamin $\mathrm{K}$ with warfarin. Journal of Clinical Pharmacy and Therapeutics. 2014; 39(1): 56-60.

5. DALPIAZ J, et al. Quality of life of warfarin users treated at the public health system. R Epidemiol Control de Infec. 2017; 7(3): 181-8.

6. DUTRA DD, et al. Cardiovascular disease and associated factors in adults and elderly registered in a basic health unit. J. res.: fundam. care. online. 2016; 8(2): 4501-09.

7. FERREIRA J, MIRCO A. Systematic review of costeffectiveness analyses of novel oral anticoagulants for stroke prevention in atrial fibrillation. Rev Port Cardiol. 2015; 34(3): 179-91.

8. FIGUEIRÊDO TR, et al. Perfil sociodemográfico e clínico de pacientes em tratamento com anticoagulantes orais. Revista da Rev Rene. 2017; 18(6): 742-8.

9. LEAL PM, et al. Construindo soluções para segurança do paciente cardiopata em uso de varfarina: estudo qualitativo. Texto contexto - enferm. 2020; 29:e 20180002: 1-12.

10. MALTA DC, et al. Doenças crônicas não transmissíveis na revista ciência \& saúde coletiva: um estudo bibliométrico. Cienc Saúde Colet. 2020; 25(12): 47-57-69.

11. MALTA MM. Qualidade de vida em pacientes anticoagulados portadores de prótese valvar mecânica cardíaca: um estudo transversal [monografia]. Porto Alegre: Universidade Federal do Rio Grande do Sul, Escola de Enfermagem; 2015.

12. MORAIS AI, et al. NOAC versus AVK: avaliação da qualidade de vida em idosos com fibrilhação auricular nos cuidados de saúde primários. Rev Port Med Geral Fam. 2020; 36(1): 16-23.

13. NISHIMURA RA, et al. AHA/ACC Focused Update of the 2014 AHA/ACC Guideline for the Management of Patients With Valvular Heart Disease: A Report of the American College of Cardiology/American Heart Association Task Force on Practice Guidelines. J Am Coll Cardiol. 2017; 135(25):e1159-95.

14. PELEGRINO FM. Adaptação cultural e validação do instrumento Duke anticoagulation Satisfaction Scale (DASS): versão para brasileiros em uso de anticoagulação oral. Dissertação (Mestrado em Enfermagem) - Universidade de São Paulo, Escola de Enfermagem de Ribeirão Preto, Ribeirão Preto, 2009; 168 p.

15. SILVA TE, et al. Manejo cirúrgico do paciente submetido à terapia anticoagulante oral. Revista Pró-univerSUS. 2019; $10(1): 145-9$.

16. SIMONETTI SH, et al. Preditores clínicos intervenientes na adesão de usuários de anticoagulantes orais. Enferm Foco. 2019; 10(1): 02-6.

17. SOUZA TF, et al. Knowledge and information levels and adherence to oral anticoagulant therapy with warfarin in patients attending primary health care services. J Vas Bras. 2018; 17(2): 109-16.

18. STAMBLER B, SCAZZUSO F. Targenting stroke risk and improving outcomes in patients with atrial fibrillation in Latin America. São Paulo Med J. 2016; 134(6): 534-42.

19. TARASOUTCHI F, et al. Atualização das Diretrizes Brasileiras de Valvopatias: abordagem das lesões anatomicamente importantes. Arq Bras Cardiol. 2017; 09(6 Suppl 2): 1-34.

20. The WHOQOL Group. The World Health Organization quality of life assessment (WHOQOL): position paper from the World Health Organization. 1995. Disponível em: https://pubmed.ncbi.nlm.nih.gov/8560308/. Acessado em: 01 de março de 2021. 